\title{
The Konrad Zuse Internet Archive Project
}

\author{
Julian Röder, Raúl Rojas, and Hai Nguyen \\ Freie Universität Berlin, Institute of Computer Science, Berlin, Germany \\ \{julian.roeder, raul.rojas, hai.nguyen\}@fu-berlin.de \\ http://zuse.zib.de
}

\begin{abstract}
This paper provides an overview of the Konrad Zuse Internet Archive project which has been conducted from 2010 until 2013 at Freie Universität Berlin in cooperation with Deutsches Museum in Munich ${ }^{1}$. The project has been mainly concerned with digitising and publication of previously unreleased private papers of Konrad Zuse. We want to make the history of computing relevant to the general public and the archive has been extended by creating a panorama, simulations, films and an introductory encyclopaedia.
\end{abstract}

Keywords: History of Computing, Konrad Zuse, Private Papers, Early Computers, Online Archive, Repository, Digitisation, Digital Humanities, Open Access.

\section{The Project}

In the Konrad Zuse Internet Archive Project the documents of Konrad Zuse's private papers are being digitised, analysed and published online.

Konrad Zuse (1910-1995) was a German computer pioneer born in Berlin. Between 1935 to 1945 he constructed several calculating machines that have been recognized to be among the first computers worldwide. He used mechanical components and telephone relays to build binary floating-point calculators. At the same time engineers in the UK and the US developed computers with their own technology and methods. In 1949, Zuse founded a company that was one of the first commercial computer production businesses in the world. Due to WWII, for a long time only a few people knew about Zuse's work.

In 2010 - the year of Zuse's $100^{\text {th }}$ birthday - the Zuse Project was launched at Freie Universität Berlin. The project is built around a cooperation with Deutsches Museum, where the originals of the private papers have been stored. The original documents of Zuse's private papers include sketches, photographs, manuscripts, typescripts, prints, shorthand notes. Most of this material was unpublished and has been released successively on the internet in an open access online archive (see Fig. 1). The documents were digitised in high quality for long-term archiving and then the images were processed for the presentation in a web browser. With the publication of these documents the project contributes to the preservation of cultural heritage and provides

1 The project was supported by the German Research Foundation (DFG). 
public access to it. The Konrad Zuse Internet Archive is the digital primary source in the world for those who want to become acquainted with Zuse's work and study it in detail.

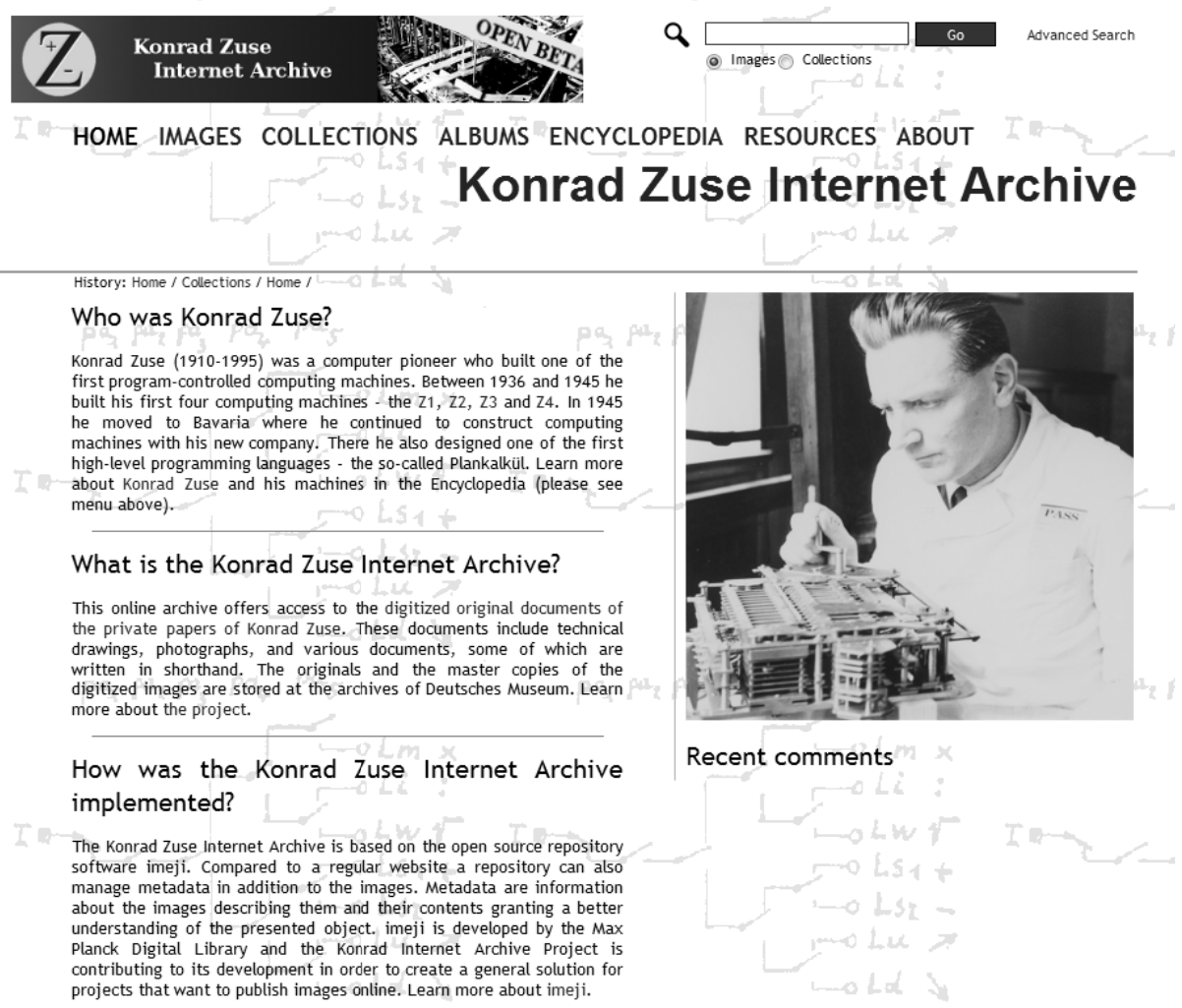

Fig. 1. Screenshot of the project website

\section{Making the History of Computing Relevant}

The goal of the project in general was to encourage people to explore Konrad Zuse's work, to learn about it, and to understand the history of computing. In order to turn information about computer history into a form that people can understand and appreciate the project took several steps which shall be described below. For some of these actions, education experts were involved to ensure the comprehensibility of the presented subjects by non-experts.

\subsection{Panorama}

In order to understand old computing machines it is important to give interested persons the opportunity to get a picture of the object in question. The first step in 
approaching complex machinery such as a computer is to become acquainted visually by grasping its dimensions, structure, materials etc. Therefore the project created a sophisticated $360^{\circ}$ object panorama of the Z1 calculating machine (Zuse's first computer, constructed in $1938^{2}$ ) consisting of over 1000 high-resolution images. The user can move around the machine, view it from different heights and zoom in to see the different elements of each functional unit (see Fig. 2). The whole machine is broken down into several functional units to illustrate the interplay of the different parts. Thus each functional unit is bordered by a white line to emphasize and separate it from the others. If a user hovers over a functional unit of the machine a description text is displayed to clarify its role. The panorama gives a good first impression of a historical computing machine and opens up the contextualizing and understanding of follow-up experiences in this field.

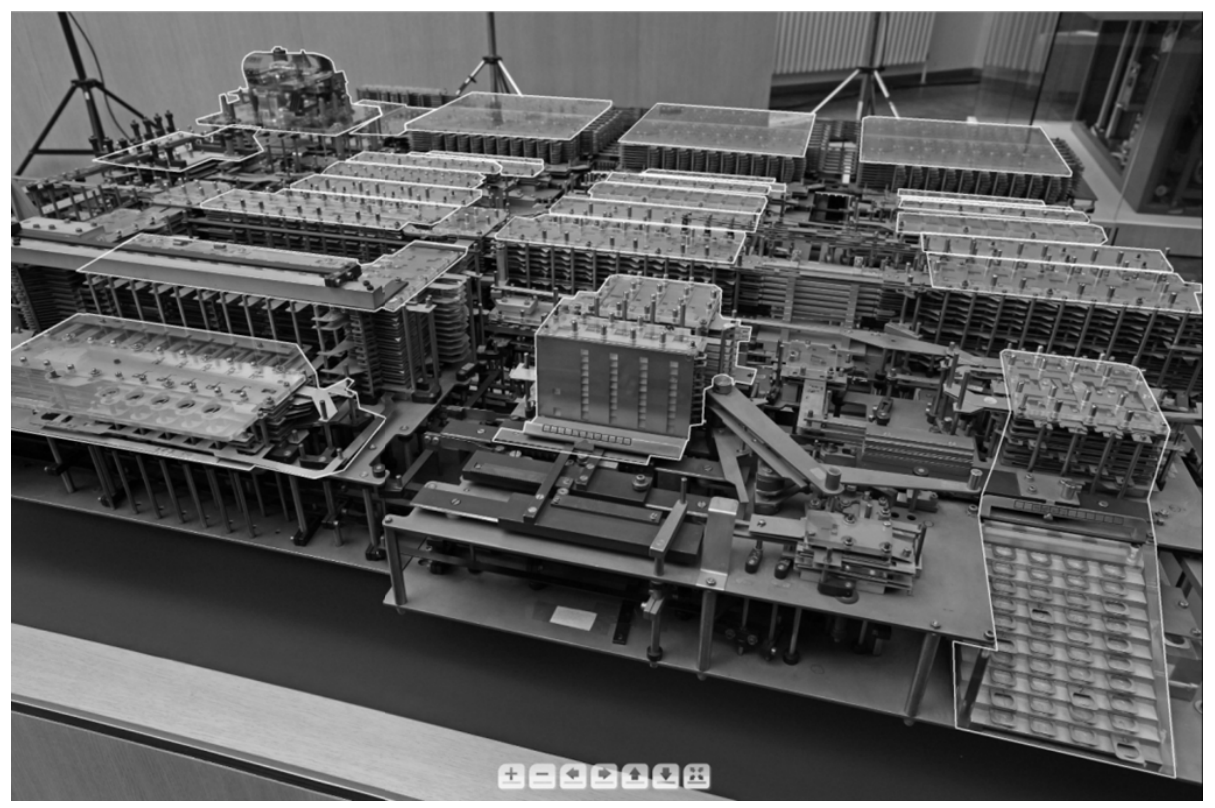

Fig. 2. Screenshot of the panorama

\subsection{Simulations}

To demonstrate the operational functionalities of Zuse's machines several simulations have been developed in the project. The interactive simulations encourage the users to explore the work of Konrad Zuse. Another crucial element is the flexible usability of the simulation. Giving the possibility to freely rotate, continuously zoom, adjust the transparency and toggle on and off the visibility of different layers of the machine at will, grants an interesting vein of discovering the key features

2 Since the original machine was destroyed during WWII, Zuse built a reconstruction in the 1980s of which the photos were taken. 
and details of the machine. The operationality of the machine is made clear by the graphical representation of each construction element and their movements. Coloured highlighting indicates where elements have been activated and how they initiate movements of other elements to bring the machine into the next state.

Another significant construction requirement was the meaningful usability of the simulation. The user can input numbers into the simulation (see Fig. 3) and afterwards the detailed calculation is simulated step by step. This means that the users can really calculate with the simulated machine. For each instructional machine step the mathematical meaning, such as intermediate results and carry-overs in binary and decimal notation, are displayed. Thus the mathematical and the engineering aspects of the machine are connected and it is spelled out how they constrain each other. Buttons to start, stop, pause, reset and repeat the last computational step of the machine, along with some expert settings, allows controlling the simulation in an individual fashion and timing. In this manner the user interaction is directly connected with the object and thereby an intensive empowerment is achieved.

Since all these functionalities may confuse non-experts a help page and a step-bystep guiding system is integrated into the simulation to break complicated processes down and provide explanatory descriptions of each step.
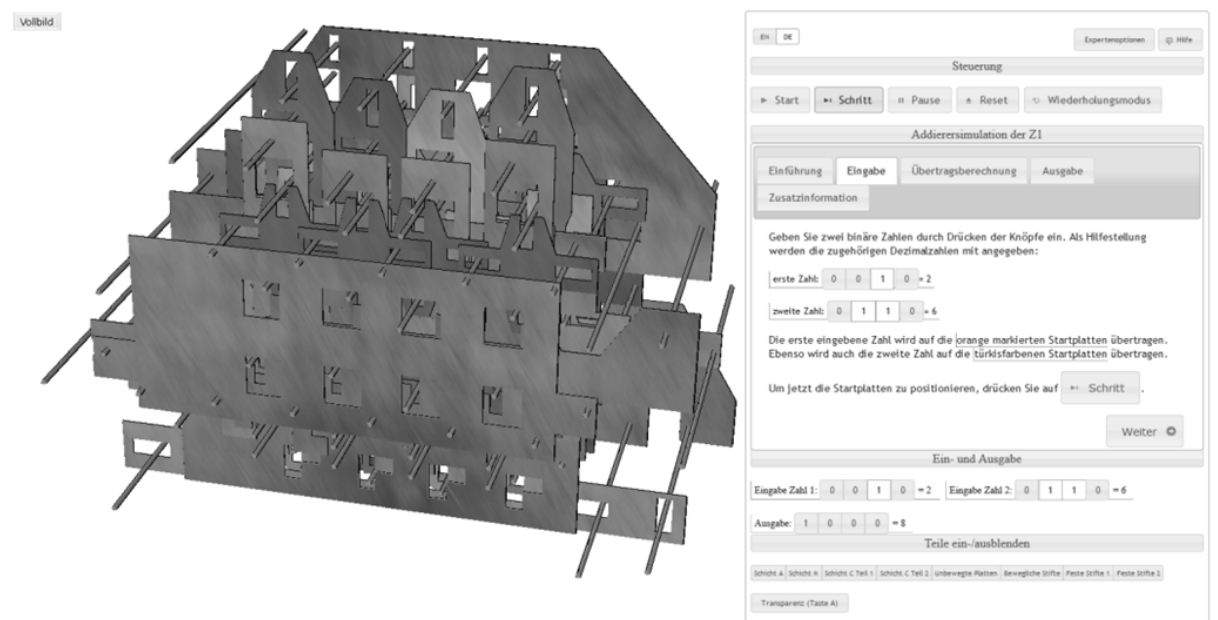

Fig. 3. Input of addends into the simulation

\subsection{Films}

Another way of presenting intricate machines is by descriptive films. On the project website two animated films about Zuse's Assembly Line Self-Replicating Systems and Helix-Tower are exhibited. These machines are not computers, but they underpin the style of engineering and way of constructional thinking of Konrad Zuse. Both machines are from the late work of Zuse and depict the continuation of his computational machine concepts to other fields of engineering. 


\section{$2.4 \quad$ Encyclopaedia}

Since Zuse's work comprises many different machines and a broad variety of employed technologies it was important to offer means to familiarize the users with certain basic terms and concepts. The technical implementation of his diverse machines and their capabilities as well as the utilized terminology within Zuse's private papers is relevant in understanding his work. The machines were made up of thousands of pieces that work together like a clock - each piece has its function and is necessary for the overall working of the machine. However Zuse used a set of standard construction elements that were reused wherever it was possible. This concept reduced the complexity of the machines and the constructional effort needed.

On the project website an encyclopaedia with thematic subpages is provided. These subpages are concerned with Konrad Zuse, his machine, essential technologies, fundamental construction elements and important theoretical and mathematical concepts. The subpages are linked to each other as well as to appropriate objects in the online archive and thus the contents can easily be reached by the users. Since the subpages are mutually explanatory a consolidated knowledge on the topic is presented. Thereby the users are enabled to put a certain object into context and to understand the function of important construction elements within Zuse's machines.

\subsection{Konrad Zuse as a Designer and Builder of Computers}

Konrad Zuse's personal history is presented on the project website. His high school and student period, the building of his first computing machines between 1936 and 1945 and the formation of his company are portrayed. Various photos of Zuse and research essays about him are available as well.

Zuse's role as an inventor of computers was worked out in detail by our cooperation partner Deutsches Museum.

\subsection{The Online Archive}

There are a lot of different possible questions about Zuse's work and in comparison to related developments in the history of computing. For this reason the goal was to create a searchable database for the digital documents (e.g. in Fig. 4). The images and the information about them (so-called metadata) are managed by an online archive software that is co-developed in the project and is integrated in the project website ${ }^{3}$.

The online archive software permits quick navigation, search and browsing and therewith easy access to the documents via (re-)entry points. Browsing through the contents is achieved by tabulated thumbnails which lead to the higher resolution images. For flexible viewing of the documents different tools for interactive viewing and scientific analysis such as zooming, rotating, panning as well as colour and brightness adjustments permits the user to examine a document in an individual manner. Thumbnail navigation, facet navigation and sortable lists grant a comfortable

3 http://zuse.zib.de 
overview over the available contents. The advanced search functionality includes logical AND, OR and NOT connectives and the possibility to specify a certain collection of images to search in. Furthermore the user can precisely define the search criteria and key words in order to obtain detailed results.

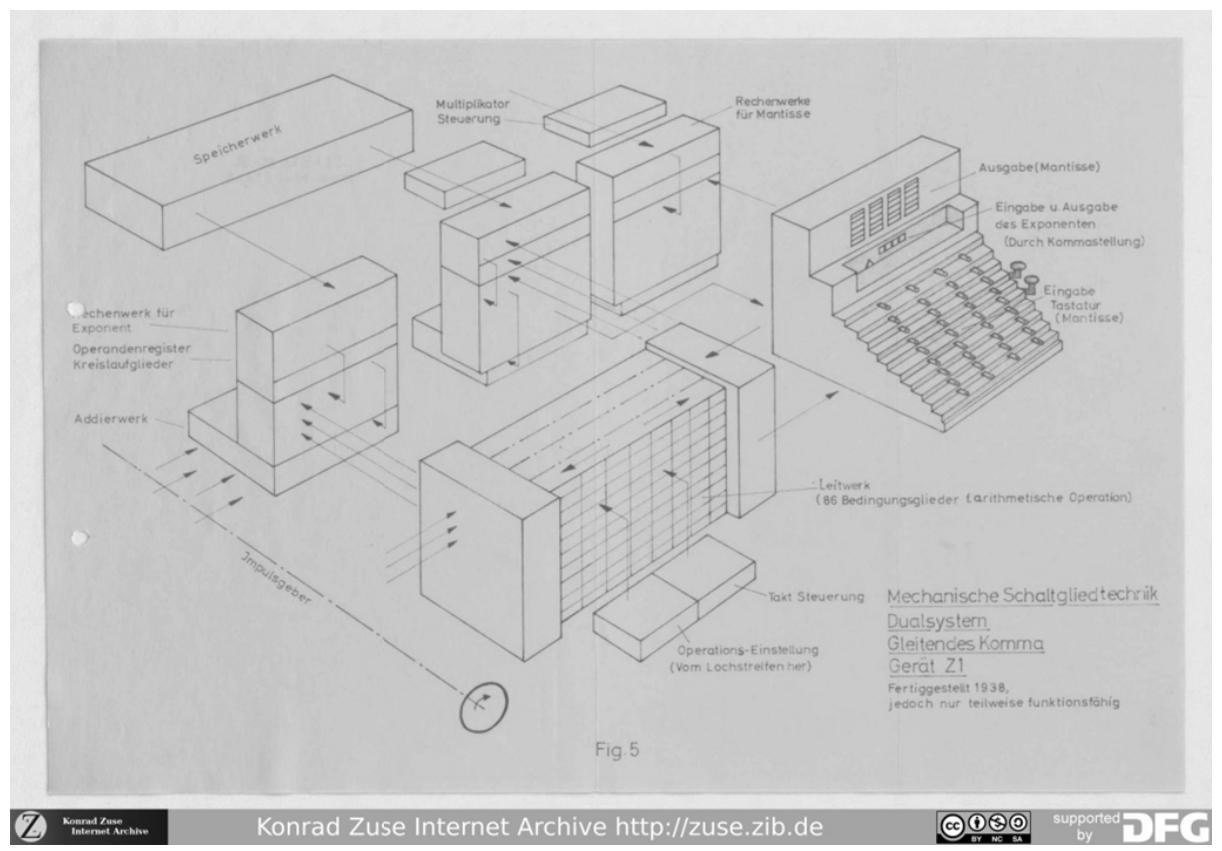

Fig. 4. Example of a document from the Konrad Zuse Internet Archive

\subsection{Open Access}

The Konrad Zuse Internet Archive is an online archive that complies with the open access paradigm. It is free of charge and all documents are published under an open license that allows reuse and sharing. No registration or login is required to view the contents. Using standard formats the stored data is compatible and interoperable with external services which can access the archive via an interface.

The online archive software is open source and is developed by a developer community in which different institutions participate.

\subsection{Relation to Modern Computers}

Computers became ubiquitous in the recent past on the one hand but opaque in their operational functionality to most users on the other. The early computers of Konrad Zuse worked with similar abstract concepts as modern computers but they are much easier to understand because they were bigger and slower. By presenting the above mentioned endeavours on the project website a better understanding of modern 
computers for non-experts shall be achieved as well. Creating a link between historical and modern computers is essential to make the history of computing interesting to the general public since it correlates history to modern everyday life.

Acknowledgements. The Konrad Zuse Internet Archive Project was supported by the German Research Foundation (DFG).

\section{References}

1. Rojas, R. (ed.): Encyclopedia of Computers and Computer History. Fitzroy Dearborn, Chicago (2001)

2. Rojas, R., Hashagen, U. (eds.): The First Computers - History and Architectures. MIT Press, Cambridge (2000)

3. Rojas, R. (ed.): Die Rechenmaschinen von Konrad Zuse. Springer, Berlin (1998)

4. Rojas, R.: How to make Konrad Zuse's Z3 a universal computer. IEEE Annals of the History of Computing 20(3), 51-54 (1998)

5. Rojas, R.: Konrad Zuse's Legacy: The Architecture of the Z1 and Z3. IEEE Annals of the History of Computing 19(2), 5-16 (1997) 